\title{
Atractylodin may induce ferroptosis of human hepatocellular carcinoma cells
}

\author{
Yongfei He ${ }^{1 \#}$, Dalang Fang ${ }^{2 \#}$, Tianyi Liang ${ }^{1}$, Hongbing Pang ${ }^{3}$, Yingdan Nong ${ }^{3}$, Libo Tang ${ }^{4}$, Ziye Yang ${ }^{4}$, \\ Chunmiao $\mathrm{Lu}^{3}$, Xiao $\mathrm{Han}^{3}$, Shuqi Zhao ${ }^{1}$, Shutian Mo ${ }^{1}$, Yuhua Meng ${ }^{5}$, Chuangye Han ${ }^{1}$, Tao Peng ${ }^{1}$ \\ ${ }^{1}$ Department of Hepatobiliary Surgery, the First Affiliated Hospital of Guangxi Medical University, Nanning, China; ${ }^{2}$ Department of Breast and \\ Thyroid Surgery, The Affiliated Hospital of Youjiang Medical University for Nationalities, Baise, China; ${ }^{3}$ Cancer Hospital affiliated to Guangxi \\ Medical University, Nanning, China; ${ }^{4}$ School of Basic Medical Sciences, Guangxi Medical University, Nanning, China; ${ }^{5}$ Department of Pathology, \\ Shunde Hospital, Southern Medical University (The First People's Hospital of Shunde, Foshan), Foshan, China \\ Contributions: (I) Conception and design: T Peng, Y He; (II) Administrative support: T Peng; (III) Provision of study materials or patients: Y He, D \\ Fang; (IV) Collection and assembly of data: T Liang, H Pang, Y Nong, L Tang, Z Yang, C Lu, X Han; (V) Data analysis and interpretation: S Zhao, \\ S Mo, Y Meng, C Han; (VI) Manuscript writing: All authors; (VII) Final approval of manuscript: All authors. \\ \#These authors contributed equally to this work. \\ Correspondence to: Tao Peng. Department of Hepatobiliary Surgery, the First Affiliated Hospital of Guangxi Medical University, Nanning, China. \\ Email: pengtaogmu@163.com.
}

Background: It has been reported that atractylodin has a potential antitumor effect. This study aimed to investigate the effects of atractylodin on Huh7 and Hccm hepatocellular carcinoma (HCC) cells and its molecular mechanism.

Methods: Huh7 and Hccm cells were cultured in vitro, and their viability was detected by CCK- 8 assay and the half inhibitory concentration (IC50) was calculated. The cells were treated with different concentrations of atractylodin, and the migration and invasion ability of cells was detected by scratch assay and Transwell assay. The cell cycle change and apoptosis rate were detected by flow cytometry. IlluminaHiSeq4000 platform was used for transcriptome sequencing, and the results were analyzed for gene differential expression, gene function, and signal pathway enrichment. Morphological changes of cells were detected by transmission electron microscopy, reactive oxygen species (ROS) levels were detected by DCFH-DA probe, and the expressions of ferroptosis related proteins GPX4, ACSL4, FTL, and TFR1 were detected by Western blot.

Results: The results showed that atractylodin could inhibit the proliferation, migration, and invasion of Huh7 and Hccm cells, regulate the cell cycle, and induce cell apoptosis and G1 phase cell cycle arrest. In addition, it could significantly induce the increase of intracellular ROS levels, decrease the expression of GPX4 and FTL proteins, and up-regulate the expression of ACSL4 and TFR1 proteins.

Conclusions: Atractylodin can inhibit the proliferation, migration, and invasion of Huh7 and Hccm liver cancer cells, and induce cell apoptosis and cell cycle arrest. In addition, our results suggest that atractylodin may induce ferroptosis in HCC cells by inhibiting the expression of GPX4 and FTL proteins, and upregulating the expression of ACSL4 and TFR1 proteins.

Keywords: Hepatocellular carcinoma (HCC); atractylodin; ferroptosis

Submitted Aug 06, 2021. Accepted for publication Sep 09, 2021.

doi: $10.21037 /$ atm-21-4386

View this article at: https://dx.doi.org/10.21037/atm-21-4386 


\section{Introduction}

Primary liver carcinoma is the third leading cause of malignant tumor-related death, of which about $80 \%$ is hepatocellular carcinoma (HCC) (1). In recent times, the early diagnosis and treatment of liver cancer have gradually improved and the mortality and morbidity of HCC has reduced, based on the strengthening of preventive surveillance of hepatitis viruses and health examinations (2). However, in many countries and regions, including China, mortality remains high because the chance of surgical resection is already lost by the time of diagnosis in many cases (3). Significant progress has recently been made in drug therapy, especially anti-angiogenic drugs combined with immunotherapy, which has resulted in an objective response rate of about $30 \%$ and median survival time of about 20 months in patients with advanced or unresectable HCC $(4,5)$. Although immune checkpoint inhibitors and targeted drugs have achieved good clinical efficacy, rash, gastroenteritis, endocrine system disease, and immunerelated adverse reactions often occur in the treatment process, and some patients may be complicated with serious reactions or even death $(6,7)$.

Recent studies have shown that Chinese medicine can participate in the whole process of anti-tumor therapy and play an important role in immunotherapy. The anti-tumor effect of Chinese medicine monomers has gradually become the focus of research at home and abroad, and several studies have shown that Shikonin inhibits the progression of HCC by regulating cell proliferation, apoptosis, glycolysis, and epithelial-mesenchymal transformation (8-10). Astragalus polysaccharide can inhibit tumor growth and increase the immune response, and in combination with codonopsis polysaccharides, can also increase the expression of peripheral $\mathrm{T}$ lymphocytes and inhibit tumor invasion and metastasis $(11,12)$. Atractylodin is a genus plant of Asteraceae and one of the effective components of atractylodes, and studies have shown that it can reduce limps-induced acute lung injury by inhibiting NOD-like receptor protein 3 (NLRP3) inflammasome and Toll-like receptor 4 (TLR4) signaling pathways (13). Yu et al. reported that atractylodin had an enhanced contractile effect on inflation-induced intestinal motility disorders (14), and could induce apoptosis of bile duct cancer cells by inducing caspase cascade, indicating that its mechanism of inhibiting the activity of these cells is similar to its anti-inflammatory mechanism (15). These reports indicate that atractylodin has great potential value in clinical application.
Ferroptosis is an iron-dependent non-apoptotic form that regulates cell death and is associated with an imbalance of intracellular REDOX homeostasis (16), while sorafenib is a commonly used first-line treatment for HCC. Studies have shown that sorafenib could induce increased lipid oxidation levels in HCC cells, resulting in cell death, which is closely related to ferroptosis (17). In addition, haloperidol, erastin, and sorafenib in combination can induce the increase of intracellular iron ions and production of many active oxygen species leading to the increase of lipid peroxidation, thereby inducing iron death in HCC cells (18). These reports suggest that iron, lipid peroxidation, and amino acid metabolism are the main regulatory mechanisms of ferroptosis, and the abundant free iron and high level of reactive oxygen species (ROS) in tumor cells provides a theoretical basis for the clinical use of ferroptosis in the treatment of tumors.

Various studies have demonstrated that atractylodin plays an anti-inflammatory role, regulates gastrointestinal motility, and plays an anti-tumor role, but its role in HCC remains unclear. Therefore, this study explored the effect and mechanism of atractylodin on human HCC cells and provided the theoretical basis for the development of its clinical application in HCC patients. We present the following article in accordance with the MDAR reporting checklist (available at https://dx.doi.org/10.21037/atm-21-4386).

\section{Methods}

\section{Cell culture}

Huh7 cell lines were purchased from the Chinese Academy of Sciences (CAS) Shanghai Cell Bank and were sourced from the American Type Culture Collection (ATCC). The $\mathrm{Hccm}$ cell line was derived from a 34-year-old Japanese patient who was hepatitis B surface antigen positive $(19,20)$. Cells were cultured with $10 \%$ fetal bovine serum (GibcoLife Technologies, Carlsbad, CA, USA) and 1\% penicillin/ streptomycin (P1400, Solarbio, China) Dulbecco's Modified Eagle's Medium (DMEM; BI, 06-1055-57-1A) in DMEM medium, and cultured in a $37{ }^{\circ} \mathrm{C}$ incubator with $5 \% \mathrm{CO}_{2}$.

\section{Reagents and antibodies}

Atractylodin $(\mathrm{C} 13 \mathrm{H} 10 \mathrm{O})$, purity $\geq 99 \%$, relative molecular weight: GPX4 Polyclonal Antibody (\#14432-1-AP), ACSL4 Monoclonal Antibody (\#66617-1-IG), CD71, GPX4 Polyclonal Antibody (\#14432-1-AP), ACSL4 Monoclonal 
Antibody (\#66617-1-IG), CD71 Polyclonal Antibody (\#10084-2-AP), Ferritin Light Chain Polyclonal Antibody (\#10727-1-AP), HRP-conjugated Affinipure Goat Anti-rabbit $\operatorname{IgG}(\mathrm{H}+\mathrm{L})$ (\#SA00001-2) and HRP-Conjugated Affinipure Goat Anti-Mouse IgG(H+L) (\#SA00001-1), analyzed with Cell Cycle and an Apoptosis Analysis Kit (\#C1052) purchased from Proteintech Group and Beyotime Biotechnology.

\section{Cell viability assay}

A Cell Counting Kit-8 (Biosharp) was used to detect the degree of cytotoxicity of atractylodin on Huh7 and Hccm cells, and each group was repeated three times. The cell density was adjusted to $5 \times 10^{4}$ cells $/ \mathrm{mL}, 100 \mu \mathrm{L}$ was added to a 96-well plate, cultured at $37{ }^{\circ} \mathrm{C}$ in a $5 \% \mathrm{CO}_{2}$ incubator overnight for 24 hours, and then treated with different concentrations of atractylodin for intervention. Ten $\mu \mathrm{L}$ CCK8 was added at different detection time points and incubated for $2 \mathrm{~h}$. The optical density (OD) was read with an absorbance value of $450 \mathrm{~nm}$ on the microplate reader. The semicirculated inhibitory concentration (IC50) of acetylides at lodges on each cell was calculated by GraphPad Prism 8, and the proliferation curve was made.

\section{Wound-bealing invasion assay}

Huh7 and Hccm cells were digested and inoculated on 6-well plates, and according to their calculated IC50, were divided into a control group, atractylodin $\mathrm{L}$ group $(5 \mu \mathrm{M} / \mathrm{L})$, atractylodin $M$ group $(10 \mu \mathrm{M} / \mathrm{L})$, and atractylodin $H$ group $(20 \mu \mathrm{M} / \mathrm{L})$. When the cells reached $80 \%$ confluence, they were vertically crossed with a sterile $100 \mu \mathrm{L}$ plastic suction head and washed with phosphate-buffered saline (PBS) three times. After washing, 2\% FBS DMEM medium containing different concentrations of atractylodin was added in groups, and photographs were taken at 0 and 24 hours under an inverted microscope (Olympus). The Transwell chamber $(8 \mu \mathrm{m}$ aperture, BD) was coated with $100 \mu \mathrm{L}$ Matrigel $(285 \mu \mathrm{g} / \mathrm{mL}$, Corning) and then placed in an incubator at $37^{\circ} \mathrm{C}$ for $1 \mathrm{~h}$ to gelatinize. A $300 \mu \mathrm{L}$ non-serum medium containing $5 \times 10^{4}$ cells with the corresponding concentration of atractylodin was then placed in each chamber. At the same time, a $700 \mu \mathrm{L}$ medium containing $10 \%$ FBS was added to each well of the 24-well plates and the Transwell chambers were placed in the 24-well plates. After 48 hours of culture, the upper compartment was washed with PBS to remove the upper compartment cells, which were fixed in methanol on the underside of the chamber and stained with crystal violet $(0.1 \%)$ at room temperature for $30 \mathrm{~min}$. After washing and drying, the stained cells were considered those that had invaded the lower chamber. Photographs were taken under an inverted microscope.

\section{Cell cycle analysis}

Huh7 and Hccm cells were digested, and a $2 \mathrm{~mL}$ medium containing $2 \times 10^{5}$ cells was placed in each well of a 6-well plate and cultured overnight at $37{ }^{\circ} \mathrm{C}$ in an incubator of $5 \% \mathrm{CO}_{2}$ for $24 \mathrm{~h}$. Cells in different groups were given the corresponding concentration of atractylodin and cultured for 24 hours. A Cell Cycle and Apoptosis Analysis Kit (Beyotime) was used to detect cell cycle, and flow cytometry was used to analyze its distribution.

\section{Cell apoptosis analysis}

Huh7 and Hccm cells were digested, and a $2 \mathrm{~mL}$ medium containing $2 \times 10^{5}$ cells was placed in each well of a 6 -well plate and cultured overnight at $37^{\circ} \mathrm{C}$ in an incubator of $5 \% \mathrm{CO}_{2}$ for $24 \mathrm{~h}$. Cells in different groups were given the corresponding concentration of atractylodin and cultured for 24 hours. A Cell Cycle and Apoptosis Analysis Kit (Beyotime) was used to detect cell apoptosis, and flow cytometry was used to analyze its distribution.

\section{$R N A-s e q$}

Huh7 cells were digested, and the cell density was adjusted to $\left(2 \times 10^{5}\right) / \mathrm{mL}$ and inoculated in 6-well plates with $2 \mathrm{~mL}$ cell suspension per well. The cells were cultured in a DMEM medium for 24 hours. The blank control group and test group $(10 \mu \mathrm{M} / \mathrm{L})$ were set up, and each group was repeated with three samples. Cells were collected 24 hours after treatment and RNA was extracted by Trizol method, and the concentration and purity were detected. The mRNAs with polyA tail were enriched by Oligo(dT) beads, and then randomly fragmented by divalent cations in fragmentation buffer. The first strand of cDNA was synthesized in the $\mathrm{M}-\mathrm{MulV}$ reverse transcriptase system by using the fragments of mRNA as a template and random oligonucleotides as primers, and the RNA strand was then degraded by $\mathrm{RNaseH}$. The second strand of cDNA was synthesized in the DNA polymerase I system using dNTPs as raw material, and after end repair, a tail was added to the purified doublestrand cDNA and the sequencing connector was connected. 
AMPure XP beads were used to screen the 370-420 bp cDNA for PCR amplification, then AMPure XP Beads were used to purify the PCR products to finally obtain the library. After library construction, the IlluminaHiSeq4000 platform was used for transcriptome sequencing.

\section{Transmission electron microscope (TEM)}

Huh7 cells in the logarithmic growth phase were inoculated in 6-well plates with $2 \mathrm{~mL}$ cell suspension per well and cultured in DMEM medium for 24 hours. The blank control group and $20 \mu \mathrm{M} / \mathrm{L}$ group were added to the culture medium containing different concentrations of atractylodin for $24 \mathrm{~h}$. The cells were digested by trypsin without EDTA and collected, and samples were prepared after fixation with $2.5 \%$ glutaraldehyde and observed under TEM (JEM-1200EX, Japan).

\section{Intracellular ROS assay}

Huh7 and Hccm cells were digested, and a $1 \mathrm{~mL}$ medium containing $1 \times 10^{6}$ cells was placed in each well of a 6-well plate and cultured overnight at $37^{\circ} \mathrm{C}$ in an incubator of $5 \% \mathrm{CO}_{2}$ for $24 \mathrm{~h}$. DMEM complete media containing different concentrations of atractylodin were added to the cell plates for $24 \mathrm{~h}$ according to the groups, and treatment was performed according to the Reactive Oxygen Species Assay Kit (Beyotime). Observation and photography were taken under an inverted fluorescence microscope, and the fluorescence intensity was calculated and analyzed using Image J software.

\section{Western blotting analysis}

Huh7 and Hccm cells were treated in groups for 48 hours, and lysates, PSMF, and protein phosphatase inhibitors were added to extract proteins. Protein concentration was measured using BCA, and protein loading buffer was added in a ratio of $4: 1$ and mixed. The proteins were separated by $10 \%$ SDSPAGE and then transferred to the PVDF membrane, which was closed with $5 \%$ skim milk for 1 hour and incubated overnight with a primary antibody at $4{ }^{\circ} \mathrm{C}$. The membrane was then incubated with the secondary antibody at room temperature for 1 hour. The bio-rad ChemiDoc MP system was used to detect and analyze the western blot results.

\section{Statistical analysis}

The results were analyzed by GraphPad Prism 8 software.
All data are represented as mean \pm standard deviation $(n=3)$, and one-way analysis of variance (ANOVA) was used to compare differences between the experimental and control groups. We used edgeR software for the analysis of the quantity of gene expression differences between groups using FDR and $\log _{2} \mathrm{FC}$ genetic variations to filter, filter condition for FDR $\leq 0.05$, and $\mid \log _{2}$ FoldChange I $>0$. For GO/pathway enrichment analysis of differential genes, cluster profile software was used for GO and DO function enrichment analysis of differential genes and KEGG pathway enrichment analysis. FDR $\leq 0.01$ was set as the threshold for gene function and enrichment analysis of signal pathways, and LPBM was tested and corrected. $\mathrm{P}<0.05$ was considered statistically significant.

\section{Results}

\section{Effect of atractylodin on cell proliferation}

CCK8 assay showed that atractylodin significantly inhibited the proliferation of Huh7 and Hccm cells compared with the control group and showed a positive correlation with the concentration. After 24 hours of atractylodin intervention, the IC50 of Huh7 cells was $22.36 \mu \mathrm{M}$ and that of Hccm was $59.71 \mu \mathrm{M}$ (Figure 1). Huh7 and Hccm cells were treated at 5, 10, and $20 \mu \mathrm{M}$ (as L, M, and $\mathrm{H}$ concentrations) for subsequent experimental treatment.

\section{Effects of atractylodin on cell migration and invasion}

The effect of atractylodin on cell migration was examined by scratch. The results showed that the migration ability of Huh7 and Hccm cells were significantly inhibited, and their migration ability gradually decreased with the increase in concentration of atractylodin (Figure 2A-2D). The effect of atractylodin on cell invasion was detected by Transwell based on Matrigel, and the results showed that the infiltrating cells in the atractylodin group were significantly reduced and positively correlated with the concentration (Figure 2E-2H). These results suggest that atractylodin can reduce the invasiveness of Huh7 and Hccm cells.

\section{Effect of atractylodin on cell cycle}

Cell cycle test showed that with the increase in concentration of atractylodin, the number of Huh7 cells in the G1 phase gradually increased, and the number of CELLS in the S/G2 phase gradually decreased, and the same performance was 

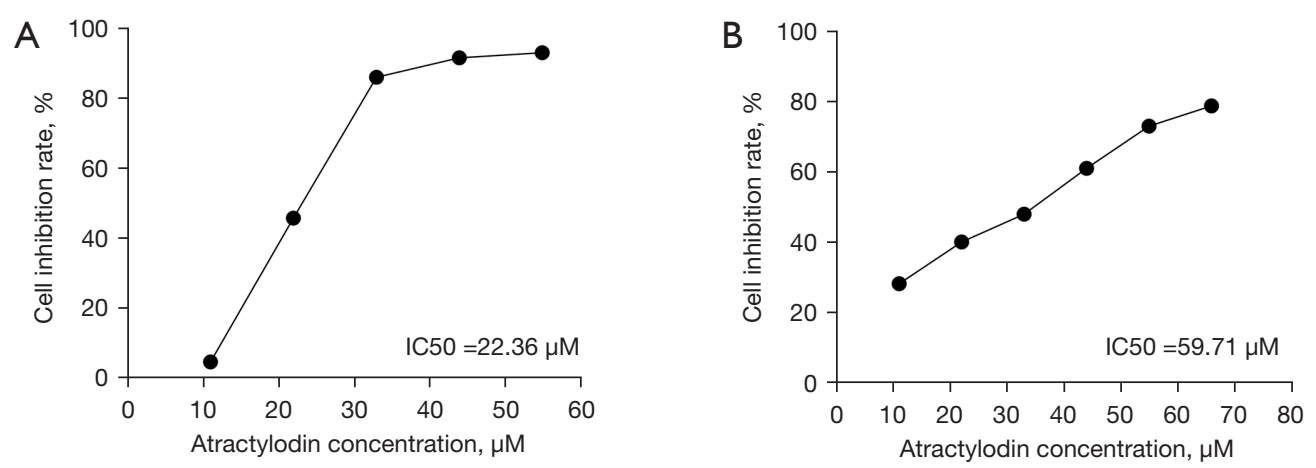

Figure 1 Effect of atractylodin on the proliferation of Huh7 and Hccm cells. (A) Huh7 cells were treated with different concentrations of atractylodin for 24 hours, and the inhibition rate of the cells was calculated. IC50 was $22.36 \mu M$. (B) Hccm cells were treated with different concentrations of atractylodin for $24 \mathrm{~h}$, and the inhibition rate of Hccm cells was calculated. IC50 was $59.71 \mu \mathrm{M}$.

shown in Hccm cells (Figure 3). These results showed that atractylodin significantly induced cell cycle arrest in the G1 phase.

\section{Effect of atractylodin on cell apoptosis}

Apoptosis detection showed that atractylodin could significantly increase the apoptosis rate compared with the control group, but with the increase of concentration, the apoptosis rate did not significantly increase (Figure 4).

\section{Screening of differentially expressed genes in cells treated with atractylodin}

A total of 3,622 differentially expressed genes were screened, among which 1,497 genes were significantly up-regulated, and 2,125 genes were significantly down-regulated $(\mathrm{P}<0.05)$ (Figure $5 A$ ). The results showed that atractylodin had a significant effect on Huh7 transcriptome expression.

\section{Differential gene function and signal patbway enrichment analysis}

Enrichment analysis of gene function of the control group and experimental group, using FDR for inspection with $\mathrm{P}$ value correction and FDR $<0.05$ as the threshold, showed a total of 458 genes by the screening of biological process, and significantly enriched the biological function of main substances such as amino acids, cholesterol metabolism, biological control, cell growth, protein translation, catalytic activity, and signal transduction . KEGG analysis showed that the differentially expressed genes were mainly concentrated in the ferroptosis, ECM-receptor interaction, cholesterol metabolism, and PI3K-Akt pathways (Figure 5B-5C).

\section{Effect of atractylodin on cell morphology}

The effects of atractylodin on Huh7 cells was observed by cell electron microscopy, and the results showed that mitochondria were small and atrophied, the mitochondrial crest was reduced or even disappeared, the membrane density proliferated, and the nuclear morphology was normal, in line with the morphological characteristics of ferroptosis (Figure 6).

\section{Effects of atractylodin on ROS levels in cells}

A Reactive Oxygen Species Assay Kit was used to detect the ROS level in cells, and the results showed that these were significantly increased with atractylodin, and that this increase was drug concentration dependent (Figure 7).

\section{Changes to atractylodin in ferroptosis protein levels}

Western blot results showed that, compared with the control group, atractylodin decreased the protein expressions of GPX4 and FTL in Huh7 and Hccm cells, and up-regulated the protein expressions of ACSL4 and TFR1 (Figure 8).

\section{Discussion}

HCC is a global health problem. As drugs currently used to treat HCC have many adverse reactions in clinical practice, there is a need to establish less toxic methods to treat 

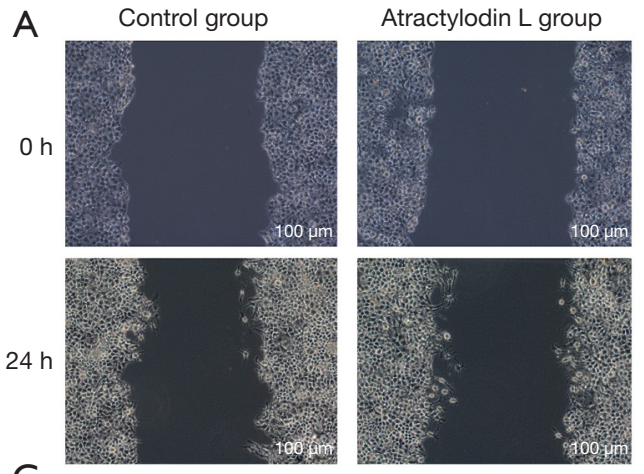

C
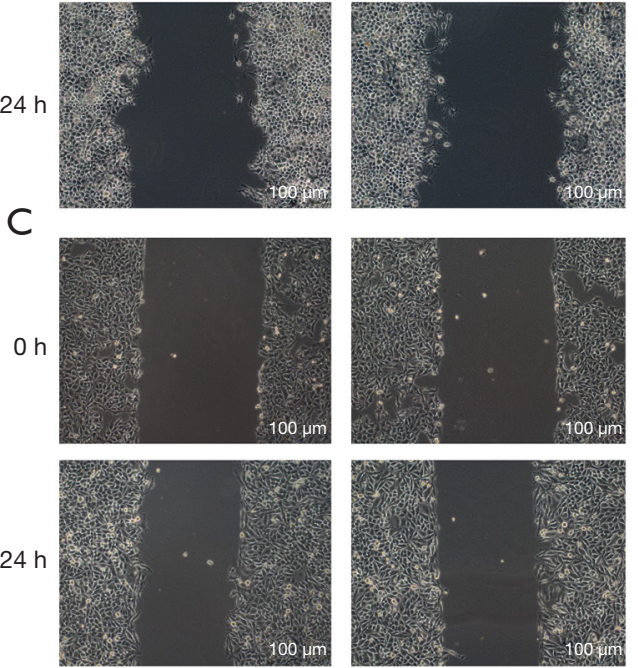

E

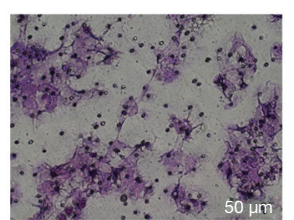

G

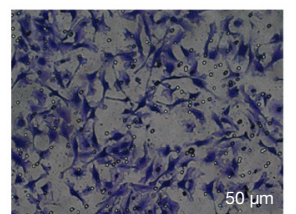

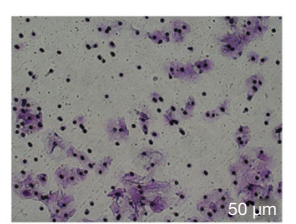

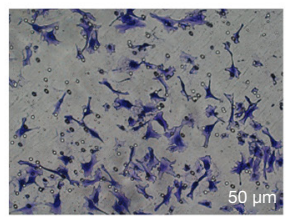

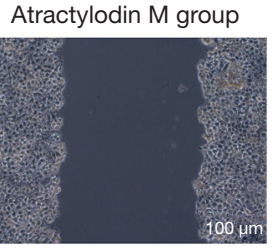
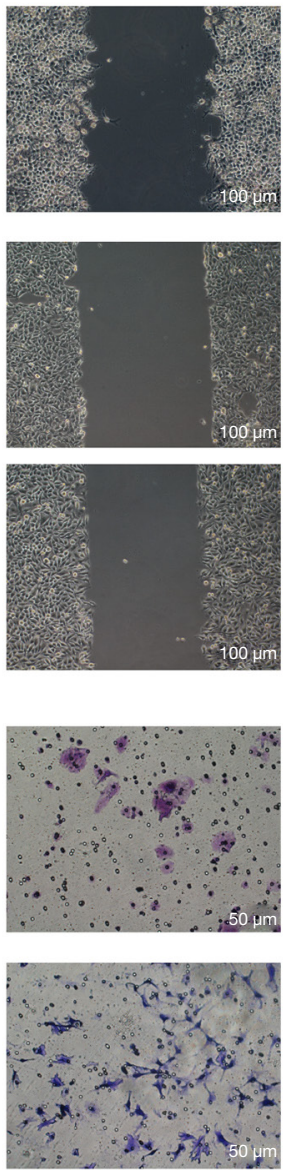
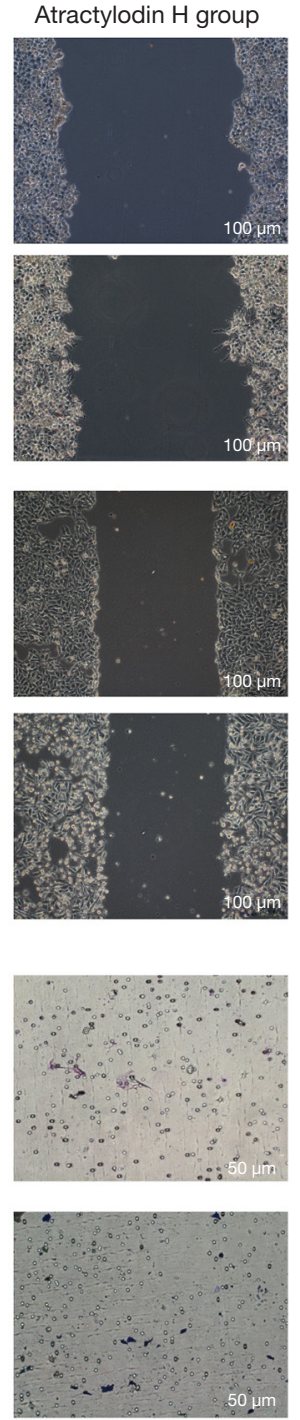

B

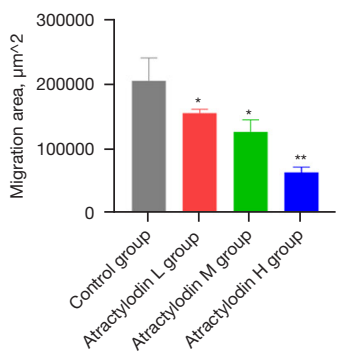

D

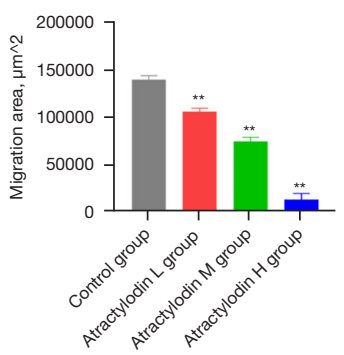

$\mathrm{F}$

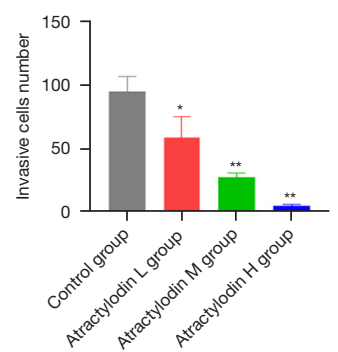

$\mathrm{H}$

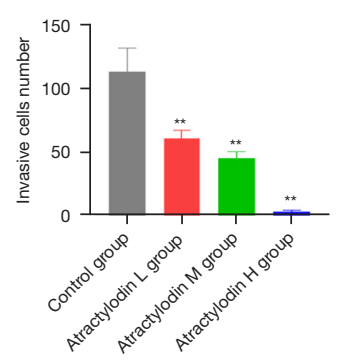

Figure 2 Effects of atractylodin on migration and invasion of Huh7 and Hccm cells. (A-D) Were the migration area of Huh7 and Hccm cells treated with atractylodin for 24 hours. (E-H) Were used to detect the number of invasive Huh7 and Hccm cells treated with atractylodin for 24 hours by Transwell assay, use crystal violet staining. Data are expressed as mean of three trials $\pm \mathrm{SD} .{ }^{*}, \mathrm{P}<0.05 ;{ }^{* *}, \mathrm{P}<0.01$, atractylodin group versus control group. Atractylodes L group: $5 \mu \mathrm{M}$; atractylodes $\mathrm{M}$ group: $10 \mu \mathrm{M}$; atractylodes $\mathrm{H}$ group: $20 \mu \mathrm{M}$.

the disease. The clinical practice of treating tumors with traditional Chinese medicine has a long history in China, and the extraction of effective anticancer active ingredients from plants has attracted more and more attention from scholars, playing an important role in alleviating the toxic and side effects of chemoradiotherapy, delaying survival, and improving survival rates $(21,22)$. Studies have reported that atractylodin inhibited bile duct cancer cell migration and induced autophagy through the PI3K/AKT/mTOR and p38MAPK signaling pathways (23), and could significantly improved the survival rate of tumor-bearing rats (24). At present, the effect of atractylodin on HCC has not been reported. In our study, we proved that atractylodin can inhibit the proliferation, migration, invasion, and promote 

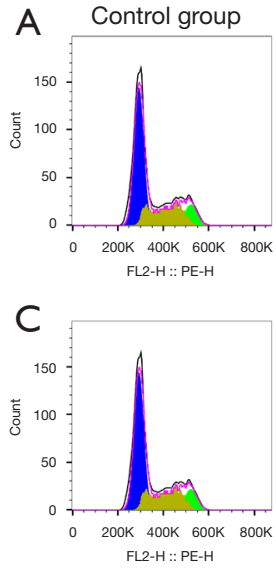
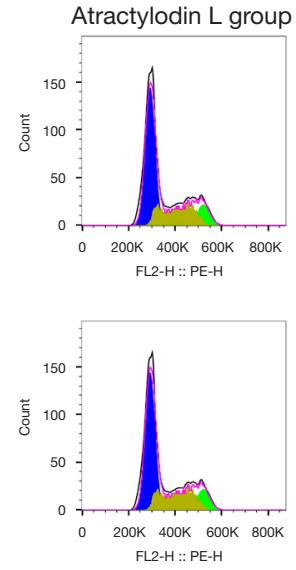
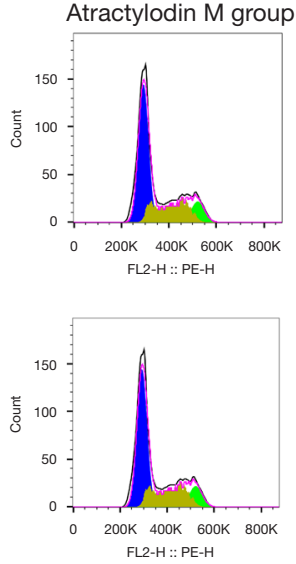
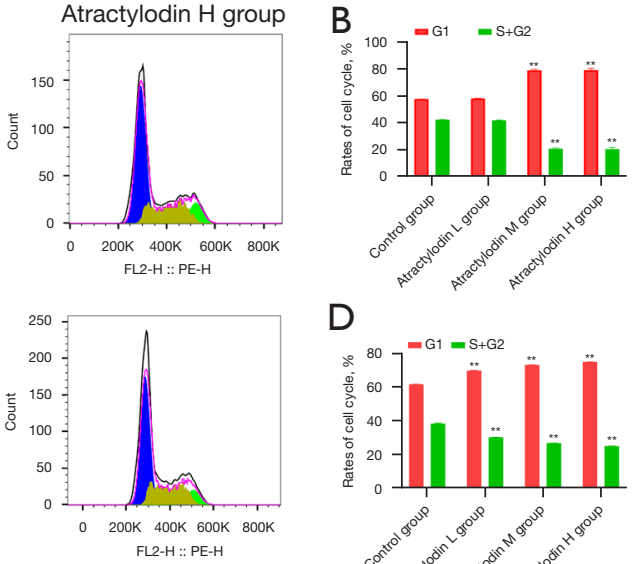

D

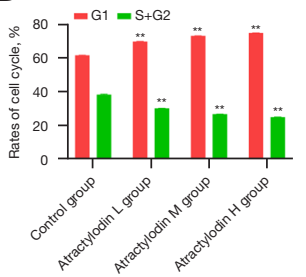

Figure 3 Effects of atractylodin on Huh7 and Hccm cell cycle. (A,B) Are the effects of atractylodin on the Huh7 cell cycle. (C,D) Are the effects of atractylodes on Hccm cell cycle. Rhizoma atractylodin contrast group, control group with different concentration, and contrast group, ${ }^{* *}, \mathrm{P}<0.01$.
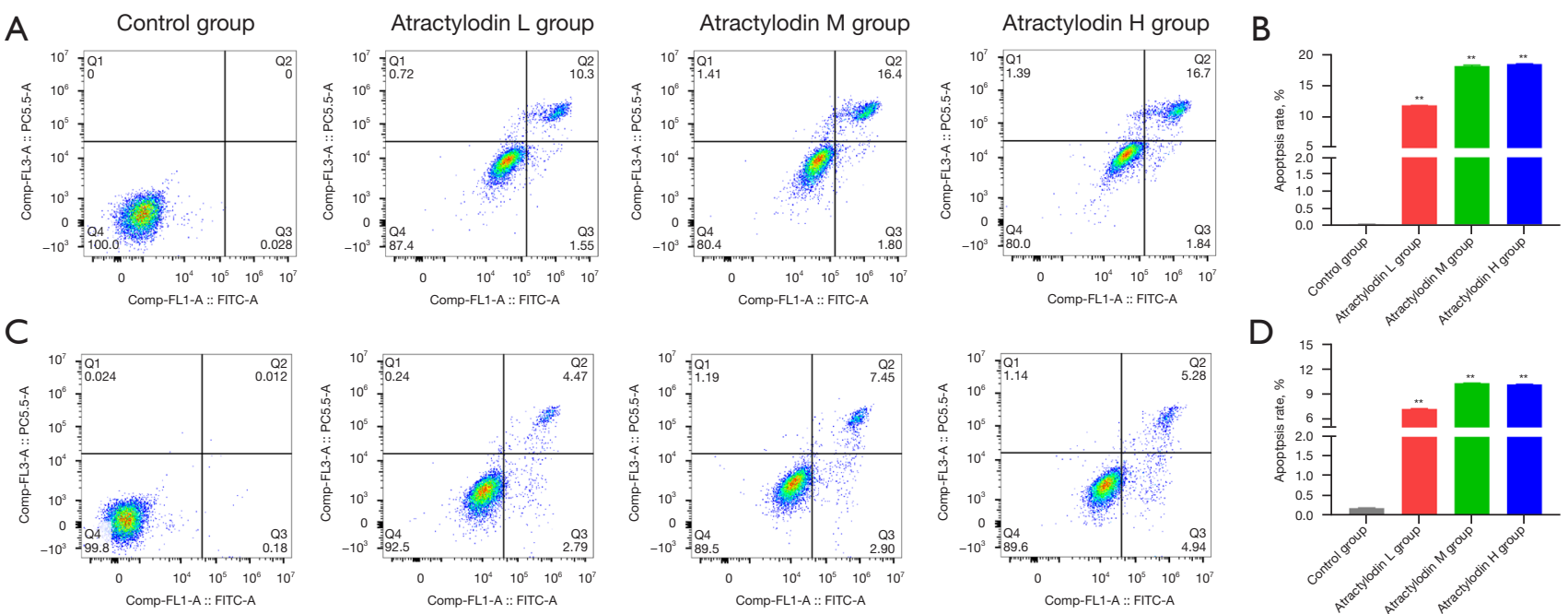

Figure 4 Effects of atractylodin on apoptosis of Huh7 and Hccm cells. (A,B) Are the effects of atractylodin on apoptosis of Huh7 cells. (C,D) Are the effects of atractylodin on apoptosis of Hccm cells. Rhizoma atractylodin contrast group, control group with different concentration, and contrast group, ${ }^{* *}, \mathrm{P}<0.01$.

apoptosis of liver cancer cells in vitro, preliminarily proving that atractylodin can kill liver cancer cells. Further studies found that atractylodin could increase the intracellular ROS level and induce ferroptosis in liver cancer cells.

The regulation of the cell cycle to inhibit cell proliferation is an important anti-tumor mechanism. The prophase (G1 phase), phase (S phase), and phase (G2 phase) of DNA synthesis are important stages of the cell cycle, during which a large amount of DNA is synthesized and replicated (25). The results of cell cycle analysis in the present study showed that atractylodin induced G1 phase stagnation in HCC cells in a concentrationdependent manner. Cell cycle arrest is also one of the main characteristics of inhibited tumor cell proliferation (26). The cell survival analysis of the present experiment also showed that cell proliferation was significantly inhibited, and the number of viable cells decreased significantly with the increase in drug concentration. Apoptosis is an 
Page 8 of 12

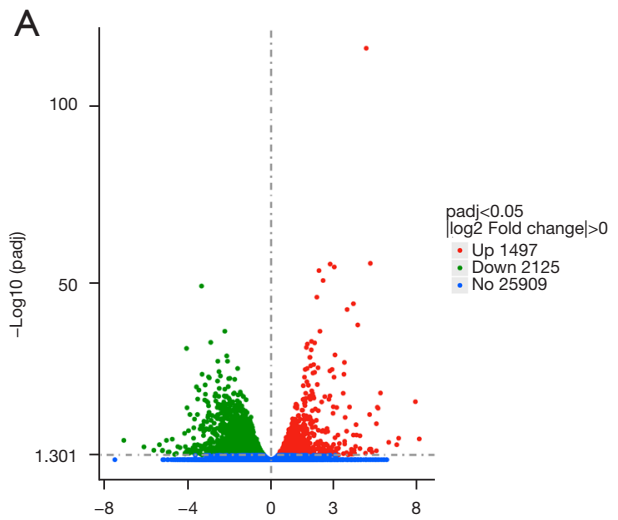

He et al. Effects of atractylodin on hepatocellular carcinoma cells

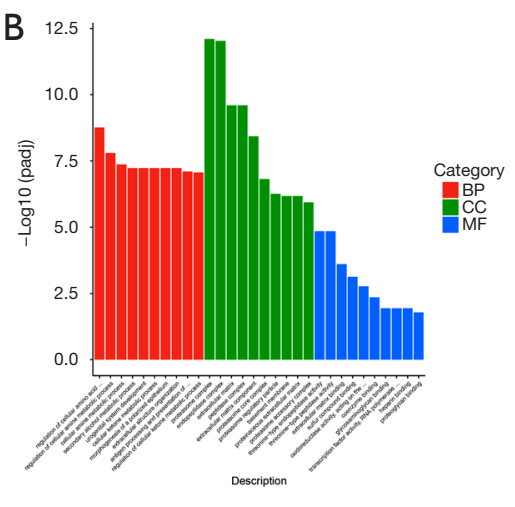

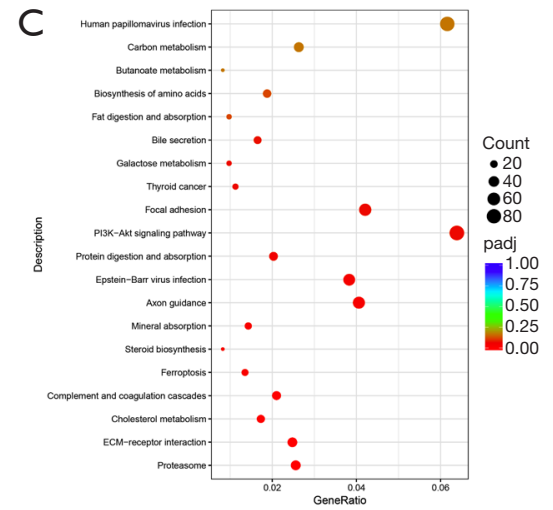

Figure 5 Effect of atractylodin on the transcriptome of Huh7 cells. (A) Differential gene volcano map where red represents a high expression of differentially expressed genes, green represents a low expression of differentially expressed genes, and blue represents no differentially expressed genes. (B) Functional enrichment of differentially expressed genes between groups. (C) Enrichment signaling pathway of differentially expressed genes between groups. BP, biological process; CC, cellular component; MF, molecular function.
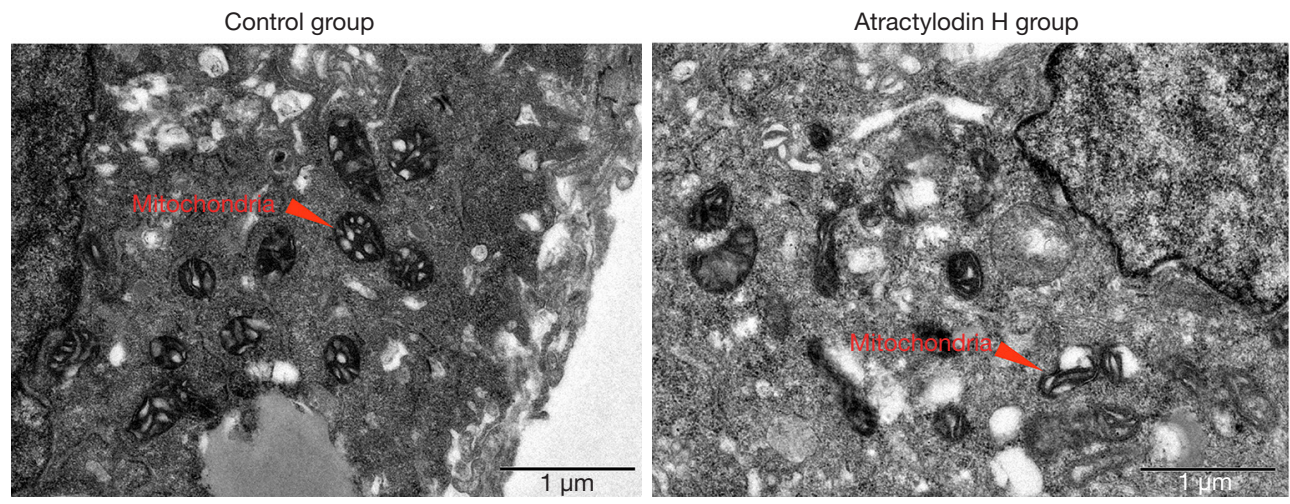

Figure 6 Effect of atractylodin on Huh7 cell morphology. TEM scanning of Huh7 cells 24 hours after atractylodin in intervention. The mitochondria are marked in red. (A) Control group. (B) High-concentration atractylodin group shows small mitochondria and atrophy, the mitochondrial crest was reduced or even disappeared, membrane density proliferation, and nuclear morphology was normal. TEM, transmission electron microscopy.

active death process regulated by genes, and its regulatory mechanism is very complex. In this study, it was found that atractylodin could significantly increase cell apoptosis, but with the increase of drug concentration, the apoptosis rate did not change significantly. Our study shows that atractylodin can effectively inhibit the proliferation of HCC cells and induce cell cycle arrest and apoptosis.

RNA-seq is a transcriptome analysis technique developed in recent years, which uses deep sequencing to provide qualitative and quantitative expression information of tissue samples (27). After drug intervention in cells or bodies, transcriptomes were fully sequenced, and the differentially expressed genes in different expression profiles were obtained through screening and comparison, to understand the target of drug action and the possible mechanism of action. Therefore, we took Huh7 cells as the sequencing object and found that atractylodin could cause significant up-regulation or down-regulation of many gene expressions in HCC cells through RNA-SEQ detection and analysis. Analysis of the functions and signaling pathways of the differentially expressed genes showed that the differentially expressed genes were mainly enriched in functions related to cell metabolism, biological regulation, and immune conduction, and mainly involving ferroptosis and ECMreceptor interaction. The cholesterol metabolism and PI3K-Akt pathway affected the metabolism, proliferation, 

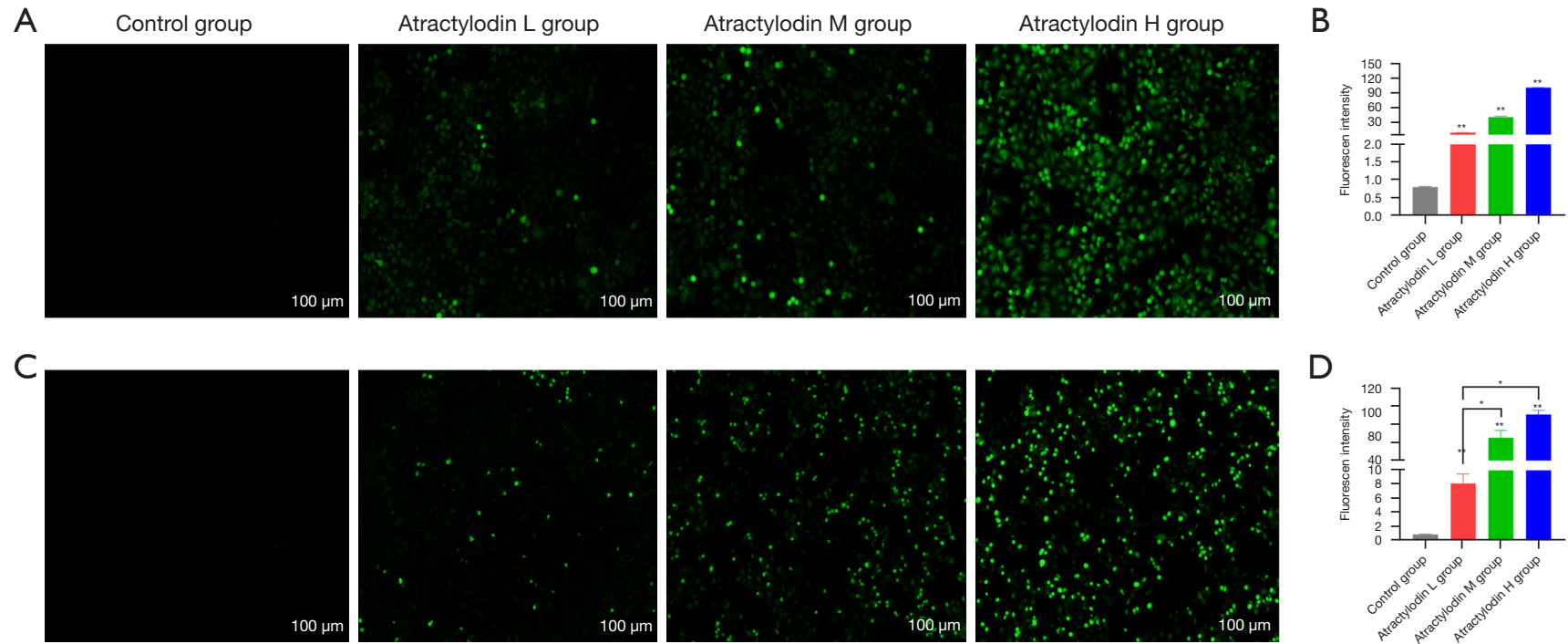

$\mathrm{D}$

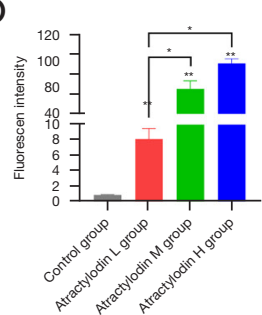

Figure 7 Effects of atractylodin on ROS levels in Huh7 and Hccm cells. (A,B) Are the effects of atractylodin on ROS in Huh7 cells. (C,D) Are the effects of atractylodin on ROS in Hccm cells. Fluorescent probe DCFH-DA loaded with reactive oxygen species kit was used to detect ROS. Rhizoma atractylodin contrast group, control group with different concentration, and contrast group, * $\mathrm{P}<0.05$; ${ }^{* *}, \mathrm{P}<0.01$. ROS, reactive oxygen species.
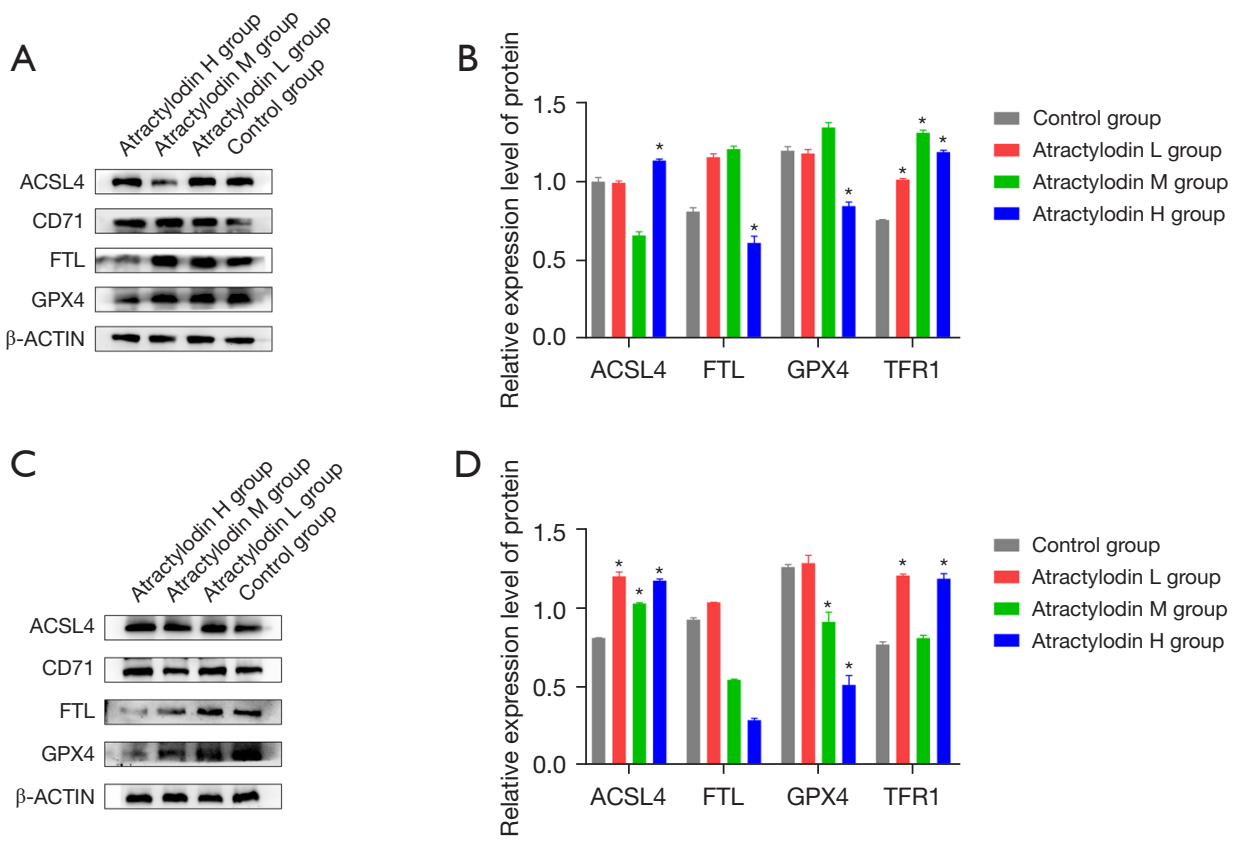

Figure 8 Effects of atractylodin on the expression of iron death-related proteins in Huh7 and Hccm cells. (A,B) Are the effects of atractylodin on the expression of ferroptosis-related proteins in Huh7 cells. (C,D) Are the effects of atractylodin on the expression of ferroptosis-related proteins in Hccm cells. Compared with the control group with different concentrations of atractylodin, ${ }^{*}, \mathrm{P}<0.05, \mathrm{CD} 71, \mathrm{TFR}$. 
apoptosis, and cycle of HCC cells.

Ferroptosis is a programmed form of death that is morphologically, biologically, and genetically distinct from necrosis and autophagy (28). Increasingly, reports have shown that iron death features such as lipid peroxides aggregation and iron metabolism disorders are found in various liver diseases to varying degrees, and regulation of ferroptosis can affect the course of the disease (29-31). It has been reported that ferroptosis is regulated by the cysteine/ glutamate transporter (system $\mathrm{X}_{\mathrm{C}}{ }^{-}$) and $\mathrm{GPX} 4$, that the effect of system $\mathrm{X}_{\mathrm{C}}{ }^{-}$or GPX4 activity can induce ferroptosis in HCC cells, and the inhibition or deletion of GPX4 can directly lead to the accumulation of lipid peroxides leading to ferroptosis in HCC cells $(32,33)$. Lipid peroxidation and iron metabolism are also closely related to the regulation of ferroptosis. The core process of iron death is the accumulation of lipid peroxidation products in cells, and iron is one of the necessary factors for the accumulation of lipid peroxides and ferroptosis (34-36). Based on the results of RNA-seq analysis, we found that the death of HCC cells caused by atractylodin may be related to ferroptosis. TEM was first performed to confirm that atractylodin could induce ferroptosis in HCC cells, and showed their mitochondria were smaller, their density increased, and their cristae decreased, which was consistent with the morphological changes of ferroptosis and preliminarily confirmed this finding. Subsequently, ROS was detected at the cellular level and found to be significantly elevated and positively correlated with concentration. GPX4 and FTL proteins associated with ferroptosis were down-regulated, while ACSL4 and TFR1 were up-regulated, which further confirmed that atractylodin could induce ferroptosis in HCC cells.

\section{Conclusions}

This study confirmed that atractylodin could effectively inhibit the proliferation, migration and invasion of HCC cells to promote apoptosis, and that ferroptosis plays a significant role in this effect. However, the exact mechanism by which this occurs requires further study.

\section{Acknowledgments}

Funding: The National Natural Science Foundation of China (Nos. 81560535, 81802874, 81072321) supported data collection and analysis, and the Natural Science Foundation of the Guangxi Province of China (Grant No.
2018 GXNSFBA138013) and Guangxi Key R\&D Program (GKEAB18221019) supported manuscript writing.

\section{Footnote}

Reporting Checklist: The authors have completed the MDAR reporting checklist. Available at https://dx.doi. org/10.21037/atm-21-4386

Data Sharing Statement: Available at https://dx.doi. org/10.21037/atm-21-4386

Conflicts of Interest: All authors have completed the ICMJE uniform disclosure form (available at https://dx.doi. org/10.21037/atm-21-4386). The authors have no conflicts of interest to declare.

Ethical Statement: The authors are accountable for all aspects of the work in ensuring that questions related to the accuracy or integrity of any part of the work are appropriately investigated and resolved.

Open Access Statement: This is an Open Access article distributed in accordance with the Creative Commons Attribution-NonCommercial-NoDerivs 4.0 International License (CC BY-NC-ND 4.0), which permits the noncommercial replication and distribution of the article with the strict proviso that no changes or edits are made and the original work is properly cited (including links to both the formal publication through the relevant DOI and the license). See: https://creativecommons.org/licenses/by-nc-nd/4.0/.

\section{References}

1. Sung H, Ferlay J, Siegel RL, et al. Global Cancer Statistics 2020: GLOBOCAN Estimates of Incidence and Mortality Worldwide for 36 Cancers in 185 Countries. CA Cancer J Clin 2021;71:209-49.

2. Yang JD, Hainaut P, Gores GJ, et al. A global view of hepatocellular carcinoma: trends, risk, prevention and management. Nat Rev Gastroenterol Hepatol 2019;16:589-604.

3. Raoul JL, Edeline J. Systemic treatment of hepatocellular carcinoma: standard of care in China and elsewhere. Lancet Oncol 2020;21:479-81.

4. Finn RS, Ikeda M, Zhu AX, et al. Phase Ib Study of Lenvatinib Plus Pembrolizumab in Patients With Unresectable Hepatocellular Carcinoma. J Clin Oncol 
2020;38:2960-70.

5. Xu J, Shen J, Gu S, et al. Camrelizumab in Combination with Apatinib in Patients with Advanced Hepatocellular Carcinoma (RESCUE): A Nonrandomized, Open-label, Phase II Trial. Clin Cancer Res 2021;27:1003-11.

6. Champiat S, Lambotte O, Barreau E, et al. Management of immune checkpoint blockade dysimmune toxicities: a collaborative position paper. Ann Oncol 2016;27:559-74.

7. Zhang S, Chen Y, Chu Q. Management strategy for adverse events of immune checkpoint inhibitors. Chinese Journal of Clinical Oncology 2018;45:609-13.

8. Hong M, Li J, Li S, et al. Acetylshikonin Sensitizes Hepatocellular Carcinoma Cells to Apoptosis through ROS-Mediated Caspase Activation. Cells 2019;8:1466.

9. Li X, Zeng X. Shikonin suppresses progression and epithelial-mesenchymal transition in hepatocellular carcinoma (HCC) cells by modulating miR-106b/SMAD7/ TGF- $\beta$ signaling pathway. Cell Biol Int 2020;44:467-76.

10. Liu T, Li S, Wu L, et al. Experimental Study of Hepatocellular Carcinoma Treatment by Shikonin Through Regulating PKM2. J Hepatocell Carcinoma 2020;7:19-31.

11. Chang WT, Lai TH, Chyan YJ, et al. Specific medicinal plant polysaccharides effectively enhance the potency of a DC-based vaccine against mouse mammary tumor metastasis. PLoS One 2015;10:e0122374.

12. Fan WT. Experimental research on the pharmacological effects of astragalus polysaccharide on immune function in mice. China Modern Medicine 2018;25:10-4.

13. Tang F, Fan K, Wang K, et al. Atractylodin attenuates lipopolysaccharide-induced acute lung injury by inhibiting NLRP3 inflammasome and TLR4 pathways. J Pharmacol Sci 2018;136:203-11.

14. Yu C, Xiong Y, Chen D, et al. Ameliorative effects of atractylodin on intestinal inflammation and co-occurring dysmotility in both constipation and diarrhea prominent rats. Korean J Physiol Pharmacol 2017;21:1-9.

15. Kotawong K, Chaijaroenkul W, Muhamad P, et al. Cytotoxic activities and effects of atractylodin and $\beta$-eudesmol on the cell cycle arrest and apoptosis on cholangiocarcinoma cell line. J Pharmacol Sci 2018;136:51-6.

16. Yang WS, Stockwell BR. Synthetic lethal screening identifies compounds activating iron-dependent, nonapoptotic cell death in oncogenic-RAS-harboring cancer cells. Chem Biol 2008;15:234-45.

17. Sun X, Ou Z, Chen R, et al. Activation of the p62Keap1-NRF2 pathway protects against ferroptosis in hepatocellular carcinoma cells. Hepatology
2016;63:173-84.

18. Bai T, Wang S, Zhao Y, et al. Haloperidol, a sigma receptor 1 antagonist, promotes ferroptosis in hepatocellular carcinoma cells. Biochem Biophys Res Commun 2017;491:919-25.

19. Watanabe T, Morizane T, Tsuchimoto K, et al. Establishment of a cell line (HCC-M) from a human hepatocellular carcinoma. Int J Cancer 1983;32:141-6.

20. Seow TK, Ong SE, Liang RC, et al. Two-dimensional electrophoresis map of the human hepatocellular carcinoma cell line, HCC-M, and identification of the separated proteins by mass spectrometry. Electrophoresis 2000;21:1787-813.

21. Zhao GS, Liu Y, Zhang Q, et al. Transarterial chemoembolization combined with Huaier granule for the treatment of primary hepatic carcinoma: Safety and efficacy. Medicine (Baltimore) 2017;96:e7589.

22. An Q, He LW, Wu Y, et al. Mechanismof Cordycepin Promotes Tumor Immunity by Regulating PD-1 Receptor of CD4 + T Lymphocytes. Journal of Nanjing University of Traditional Chinese Medicine 2018;34:495-8.

23. Acharya B, Chaijaroenkul W, Na-Bangchang K. Atractylodin inhibited the migration and induced autophagy in cholangiocarcinoma cells via PI3K/AKT/ mTOR and p38MAPK signalling pathways. J Pharm Pharmacol 2021;73:1191-200.

24. Fujitsuka N, Asakawa A, Uezono Y, et al. Potentiation of ghrelin signaling attenuates cancer anorexia-cachexia and prolongs survival. Transl Psychiatry 2011;1:e23.

25. Park JW, Choi YJ, Jang MA, et al. Chemopreventive agent resveratrol, a natural product derived from grapes, reversibly inhibits progression through $\mathrm{S}$ and $\mathrm{G} 2$ phases of the cell cycle in U937 cells. Cancer Lett 2001;163:43-9.

26. Lee DS, Lee MK, Kim JH. Curcumin induces cell cycle arrest and apoptosis in human osteosarcoma (HOS) cells. Anticancer Res 2009;29:5039-44.

27. Liu XY, Li J, Liu MM, et al. Transcriptome Profiling to Understand the Effect of Citrus Rootstocks on the Growth of 'Shatangju' Mandarin. PLoS One 2017;12:e0169897.

28. Dixon SJ, Lemberg KM, Lamprecht MR, et al. Ferroptosis: an iron-dependent form of nonapoptotic cell death. Cell 2012;149:1060-72.

29. Lörincz T, Jemnitz K, Kardon T, et al. Ferroptosis is Involved in Acetaminophen Induced Cell Death. Pathol Oncol Res 2015;21:1115-21.

30. Li X, Wang TX, Huang X, et al. Targeting ferroptosis alleviates methionine-choline deficient (MCD)-diet induced NASH by suppressing liver lipotoxicity. Liver Int 
Page 12 of 12

2020;40:1378-94.

31. Zhou Z, Ye TJ, DeCaro E, et al. Intestinal SIRT1

Deficiency Protects Mice from Ethanol-Induced Liver Injury by Mitigating Ferroptosis. Am J Pathol 2020;190:82-92.

32. Stockwell BR, Friedmann Angeli JP, Bayir H, et al. Ferroptosis: A Regulated Cell Death Nexus Linking Metabolism, Redox Biology, and Disease. Cell 2017;171:273-85.

33. Zhang X, Sui S, Wang L, et al. Inhibition of tumor propellant glutathione peroxidase 4 induces ferroptosis in cancer cells and enhances anticancer effect of cisplatin. J Cell Physiol 2020;235:3425-37.

Cite this article as: $\mathrm{He} \mathrm{Y}$, Fang $\mathrm{D}$, Liang T, Pang $\mathrm{H}$, Nong Y, Tang L, Yang Z, Lu C, Han X, Zhao S, Mo S, Meng Y, Han C, Peng T. Atractylodin may induce ferroptosis of human hepatocellular carcinoma cells. Ann Transl Med 2021;9(20):1535. doi: 10.21037/atm-21-4386

\section{He et al. Effects of atractylodin on hepatocellular carcinoma cells}

34. Louandre C, Ezzoukhry Z, Godin C, et al. Iron-dependent cell death of hepatocellular carcinoma cells exposed to sorafenib. Int J Cancer 2013;133:1732-42.

35. Yuan H, Li X, Zhang X, et al. CISD1 inhibits ferroptosis by protection against mitochondrial lipid peroxidation. Biochem Biophys Res Commun 2016;478:838-44.

36. Hernandez EP, Kusakisako K, Talactac MR, et al. Induction of intracellular ferritin expression in embryoderived Ixodes scapularis cell line (ISE6). Sci Rep 2018;8:16566.

(English Language Editor: B. Draper) 